\title{
Recombinant SjP40 protein enhances p27 promoter expression in hepatic stellate cells via an E2F1-dependent mechanism
}

\author{
Yinong Duan ${ }^{1, *}$, Lei Lyu ${ }^{1, *}$, Dandan Zhu ${ }^{1, *}$, Jianxin Wang ${ }^{2}$, Jinling Chen ${ }^{1}$, Liuting \\ Chen ${ }^{1}$, Chunzhao Yang ${ }^{1}$ and Xiaolei Sun ${ }^{1}$ \\ ${ }^{1}$ Department of Pathogen Biology, School of Medicine, Nantong University, Nantong 226001, Jiangsu, People's Republic of China \\ ${ }^{2}$ Laboratory Medicine Center, Affiliated Hospital of Nantong University, Nantong 226001, Jiangsu, People's Republic of China \\ *These authors contributed equally to this work
}

Correspondence to: Yinong Duan, email: yinongduan@aliyun.com

Keywords: liver fibrosis, P40, p27, E2F1, Schistosoma japonicum

Received: December 20, $2016 \quad$ Accepted: April 06, 2017

Published: April 19, 2017

Copyright: Duan et al. This is an open-access article distributed under the terms of the Creative Commons Attribution License 3.0 (CC BY $3.0)$, which permits unrestricted use, distribution, and reproduction in any medium, provided the original author and source are credited.

\section{ABSTRACT}

The p27 protein plays a critical role in cell cycle arrest. Our previous studies have demonstrated that recombinant P40 protein from Schistosoma japonicum (rSjP40) could induce $\mathrm{G} 1$ phase arrest of cell cycle. We, therefore, attempted to observe the effect of rSjP40 on p27 promoter activity in LX-2 cells and to explore its potential mechanisms in this study. Using both Western blot and dual-luciferase reporter assay, we demonstrated that rSjP40 could enhance the expression of p27 in LX-2 cells. Results obtained using truncated fragments of p27 promoter showed that rSjP40 increased p27 promoter activity in LX-2 cells, mainly via some transcription factors that bind to the $-1740 /-873$ region of p27 promoter. Further studies confirmed that the enhancement of p27 promoter activity induced by rSjP40 was related to E2F1 in LX-2 cells. Transfection of siRNA of E2F1 could also restore the effect of rSjP40 on expression of p27 and partially on a-SMA. Therefore, our study provided further insights into the mechanism by which rSjP40 induces LX-2 cell cycle arrest at G1 phase and inhibits HSC activation. Our results provide basis for future study of the blocking effect of rSjP40 in liver fibrosis.

\section{INTRODUCTION}

Liver fibrosis is a common pathological process observed in various chronic liver diseases [1]. During liver fibrosis, the dominant characteristic of hepatic stellate cells (HSCs) activation triggers the production of abundant extracellular matrix (ECM) proteins, including $\alpha$-smooth muscle actin ( $\alpha$-SMA) and collagen proteins [2]. Schistosome is one of the pathogenic factors that induce liver fibrosis. Sachistosome infection often leads to schistosomiasis in patients [3]. Interestingly, previous studies have shown that eggs and soluble egg antigens (SEA) from Schistosoma japonicum (S. japonicum) and Schistosoma mansoni (S. mansoni) could restrain the proliferation and activation of HSCs [4-6]. We have previously defined that recombinant $\mathrm{P} 40$ protein from $S$. japonicum (rSjP40), a dominant component of SEA, could also suppress activation of HSCs and accelerate senescence of HSCs through the STAT3/p53/p21 pathway [7].
The $\mathrm{p} 27$ protein is a structural homologue of the $\mathrm{p} 21$ protein. It has been demonstrated to play a critical role in cell cycle arrest [8]. More specifically, it has been reported that p27 is the key regulator of G0/G1 phase arrest and cell proliferation suppression in platelet-derived growth factor (PDGF)-BB-activated HSCs through repression of the PI3K/Akt/ FOXO3a pathway [9]. Since our previous studies demonstrated that SEA and its component P40 protein from $S$. japonicum could induce G1 phase arrest of cell cycle $[4,7,10]$, we aimed to further examine the effect of $\mathrm{rSjP} 40$ on the activity of $\mathrm{p} 27$ promoter in LX-2 cells and to explore its potential mechanisms in this study.

\section{RESULTS}

Expression of p27 was enhanced in $\mathrm{rSjP40}$ stimulated $L X-2$ cells

It has been demonstrated that p27 can suppress cell cycle progress through arresting cells in the G0/G1 phase by 
inhibiting the expression and activity of cyclin D1 [11, 12]. Previously, we have revealed that LX-2 cells were arrested at G1 phase after rSjP40-stimulation [7]. In this study, we further observed that $\mathrm{rSjP} 40$ could increase the protein expression level of p 27 in LX-2 cells treated with $\mathrm{rSjP} 40$ for $24 \mathrm{~h}$ and $48 \mathrm{~h}$ (Figure 1A). Results from luciferase activity analysis also confirmed that p27 promoter activities were distinctly induced in LX-2 cells stimulated with $\mathrm{rSjP} 40$ or SEA for $24 \mathrm{~h}$ (Figure 1B). These results suggested that rSjP40 could promote p27 expression in LX-2 cells.

\section{rSjP40 increased p27 promoter activity in $\mathrm{LX}-2$ cells, mainly via transcription factors that bind to $-1740 /-873$ region of the $\mathbf{p} 27$ promoter}

In order to narrow down the activity region, we established four luciferase reporter plasmids of truncated fragments of the p27 promoter, pGL3-p27a, pGL3p27b, pGL3-p27c, pGL3-p27d (Figure 2A). As shown in Figure $2 \mathrm{~B}$, transcription factors could promote p27 promoter activity by binding to the $-1740 /-1126$ region of the p27 promoter. Meanwhile, transcription factors that could inhibit p27 promoter activity may bind to the $-1126 /-873$ region of the p27 promoter (Figure $2 \mathrm{~B}$ ).

To explore the mechanism through which rSjP40 could promote p27 promoter activity, the truncated fragments were then transfected into rSjP40-stimulated LX-2 cells, respectively. Results from dual-luciferase reporter assay showed that $\mathrm{p} 27$ promoter activity was apparently increased in $\mathrm{rSjP} 40$-treated LX-2 cells transfected with pGL3-p27 and pGL3-p27a (Figure 2C) and that rSjP40 might have increased p27 promoter activity in LX-2 cells via some transcription factors that bound to the $-1740 /-873$ region of the $\mathrm{p} 27$ promoter.

\section{Transcription factor E2F1 targeted the -1740/- 873 activity region of $\mathrm{p} 27$ promoter}

Transcription factor binding sites in the -1740/873 activity region were predicted using p-match and JASPAR database. Both software tools were employed using the default settings. The prediction results of JASPAR database showed that two E2F1 binding sites $(-1447 /-1436,-1003 /-992)$, four CEBP- $\beta$ binding sites and seven STAT3 binding sites may be present in the activity region. However, results from $\mathrm{p}$-match database indicated that only one E2F1 (-1447/-1436) and one NKX2-5 binding sites are located within the $-1740 /-873$ region of $\mathrm{p} 27$ promoter. We, therefore, speculated that $\mathrm{rSjP} 40$ may promote $\mathrm{p} 27$ promoter activity in LX-2 cells through an E2F1-dependent mechanism. Furthermore, we observed that $\mathrm{rSjP} 40$ could induce E2F1 expression, but not CEBP- $\beta$ expression, in LX-2 cells by Western blot (Figure 3A). To investigate whether the two binding sites are effective, two pairs of primers were designed for CHIP. Results from CHIP experiment confirmed that E2F1 could bind to the p 27 promoter at the $-1447 /-1436$ location
(Figure 3B and Figure 3C). These results preliminarily indicated that the effect of rSjP40 on p27 promoter activity in LX-2 cells may be attributed to E2F1.

\section{rSjP40-mediated enhancement of p27 promoter activity was related to E2F1 in LX-2 cells}

Next, we explored whether the specific knockdown of endogenous E2F1 could impact p27 expression in LX-2 cells. As shown in Figure 4A, transfection with siRNA E2F1 led to reduction of $\mathrm{p} 27$ protein expression. Transfection with siRNA E2F1 also eliminated $\mathrm{rSjP} 40$-induced promotion of p27 protein expression (Figure 4B) and p27 promoter activity (Figure 4C). However, rSjP40 could not induce the activity of a mutant $\mathrm{p} 27$ promoter, which contained a mutation in the E2F1 binding site (Figure 4D). All the above results suggested that $\mathrm{rSjP} 40$ may promote $\mathrm{p} 27$ expression level in LX-2 cells via an E2F1-dependent mechanism.

\section{rSjP40-mediated inhibition of $\alpha$-SMA expression was partially related to E2F1 in LX-2 cells}

Since $\mathrm{rSjP} 40$ could inhibit $\alpha$-SMA expression in LX-2 cells [7, 13], we asked whether siRNA of endogenous E2F1 could impact $\alpha$-SMA expression in LX-2 cells. As shown in Figure 5A, $\alpha$-SMA expression could be up-regulated in rSjP40-siRNA E2F1+ group, compared to that in $\mathrm{rSjP} 40$ siRNA NC+ group $(* P<0.05)$. However, which is different from $\mathrm{p} 27$ and E2F1 expression, significant difference could be found between the rSjP40-siRNA E2F1+ group and rSjP40+siRNA E2F1+ group $\left({ }^{\#}>0.05\right)$. These results showed that transfection with siRNA E2F1 could partially reverse the inhibitory effect of $\mathrm{rSjP} 40$ on $\alpha$-SMA expression. Therefore, we concluded that $\mathrm{rSjP} 40$ may inhibit HSC activation through an E2F1/p27 pathway (Figure 5B).

\section{DISCUSSION}

Inflammatory granuloma formation and liver fibrosis are characteristics of schistosomiasis [14]. During liver fibrosis in schistosomiasis, HSC activation is one of main events of liver fibrosis, and is responsible for collagen production and $\alpha$-SMA accumulation. This developmental process of HSCs often leads to fibrogenesis in the liver of patients with schistosomiasis [15]. Hence, the inhibition of HSC activation and the induction of HSC apoptosis and senescence are often employed as the main strategies for treating liver fibrosis [16]. As previously described, cell cycle arrest at G0/G1 phase of HSCs may be an important mechanism through which potential therapeutics could block liver fibrosis. For example, An et al. have demonstrated PTEN could induce arrest at the G0/G1 and G2/M phase in both human LX-2 cells and rat primary HSCs [17]. Bohanon et al. also found that CYD0692, which is a newly designed analog of Oridonin, could induce LX-2 cell cycle arrest at S phase [18]. 
A
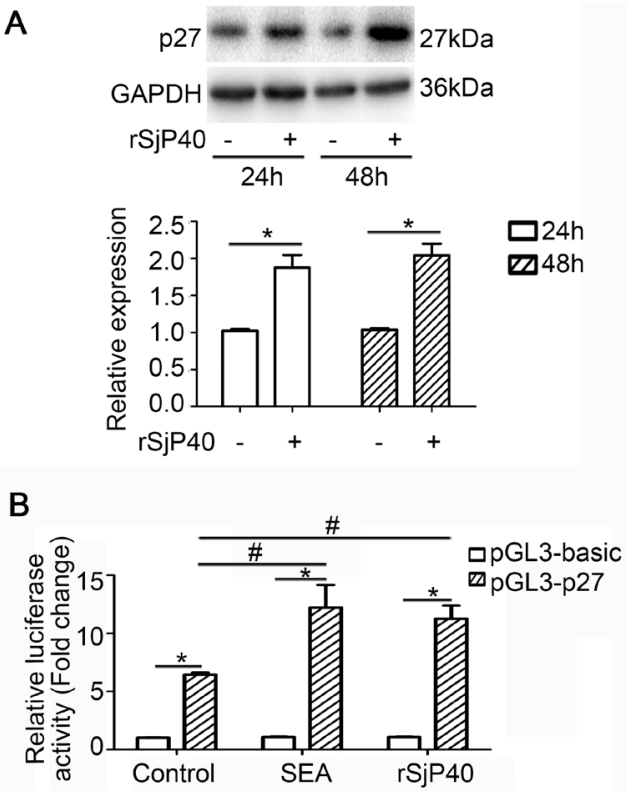

Figure 1: p27 expression was up-regulated in LX-2 cells treated with $\mathbf{r S j P 4 0 . ~ ( A ) ~ p 2 7 ~ p r o t e i n ~ e x p r e s s i o n ~ l e v e l s ~ i n ~ L X - 2 ~ c e l l s ~}$ treated with rSjP40 at the concentration of $20 \mu \mathrm{g} / \mathrm{mL}$ were evaluated by Western blot. ${ }^{*} P<0.05$, compared to each untreated group. (B) p27 promoter fluorescence activities were elevated in LX-2 cells treated with $\mathrm{rSjP} 40$ or SEA. $* P<0.05$, compared to each pGL3-basic group. ${ }^{\#} P<0.05$, compared to untreated group transfected with pGL3-p27. The data are presented as the mean \pm SEM of at least three independent experiments.

A
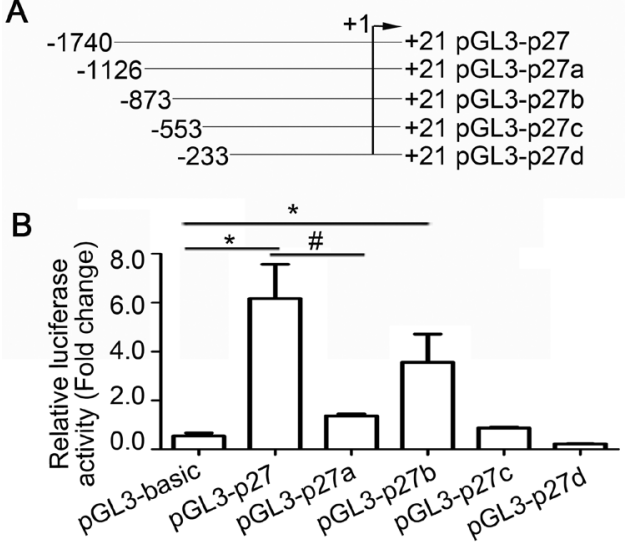

C

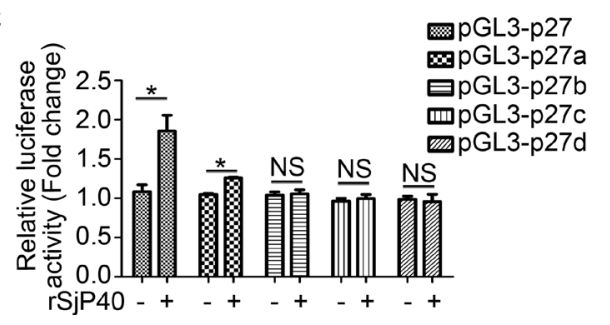

Figure 2: rSjP40 increased p27 promoter activity in LX-2 cells via transcription factors that bind to the $\mathbf{- 1 7 4 0 / - 8 7 3}$ region of the p27 promoter. (A) Diagram of the construction of p27 promoter truncated fragments. (B) Fluorescence activities of pGL3-basic, pGL3-p27, pGL3-p27a, pGL3-p27b, pGL3-p27c and pGL3-p27d in LX-2 cells were determined by dual-luciferase reporter assay. ${ }^{*} P<0.05$, compared to pGL3-basic group. ${ }^{\#} P<0.05$, compared to pGL3-p27 group. (C) LX-2 cells were treated with or without rSjP40. Fluorescence activities of pGL3-p27, pGL3-p27a, pGL3-p27b, pGL3-p27c and pGL3-p27d were determined by dual-luciferase reporter assay. ${ }^{*} P<0.05$, compared to each untreated group. NS, $P>0.05$, no significant difference was found. The data are presented as the mean \pm SEM of at least three independent experiments. 


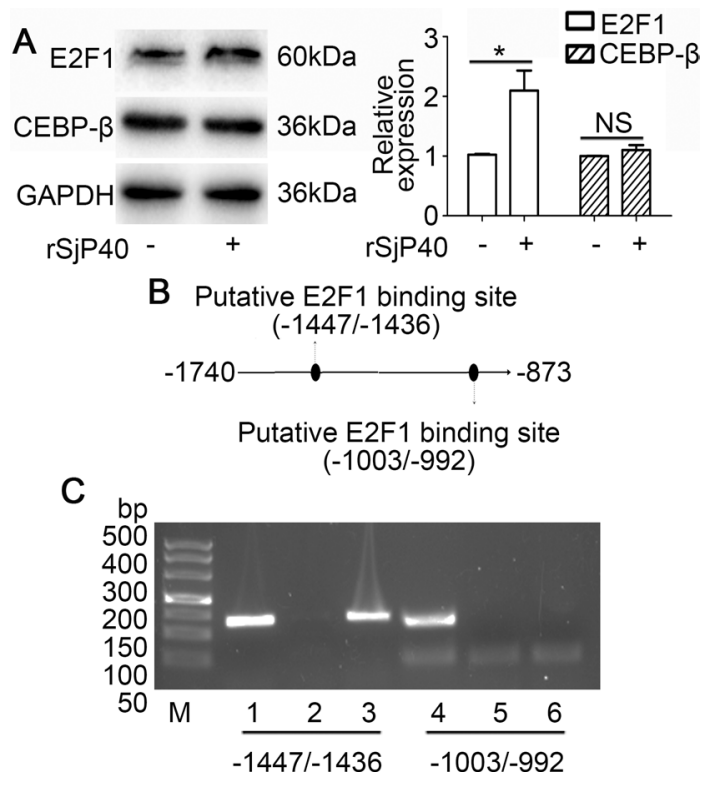

Figure 3: Transcription factor E2F1 binds to the $\mathbf{- 1 7 4 0 / - 8 7 3}$ activity region of the p27 promoter. (A) Protein expression of E2F1 and CEBP- $\beta$ after rSjP40 treatment as investigated by Western blot. ${ }^{*} P<0.05$, compared to untreated group. NS, $P>0.05$, no significant difference was found. (B) Diagram of E2F1 binding sites in the $-1740 /-873$ activity region of the p27 promoter. (C) CHIP analysis was performed to confirm the binding of E2F1 to the p27 promoter. Lanes 1 and 4: input group. Lanes 2 and 5: normal IgG group. Lanes 3 and 6: anti-E2F1 group.
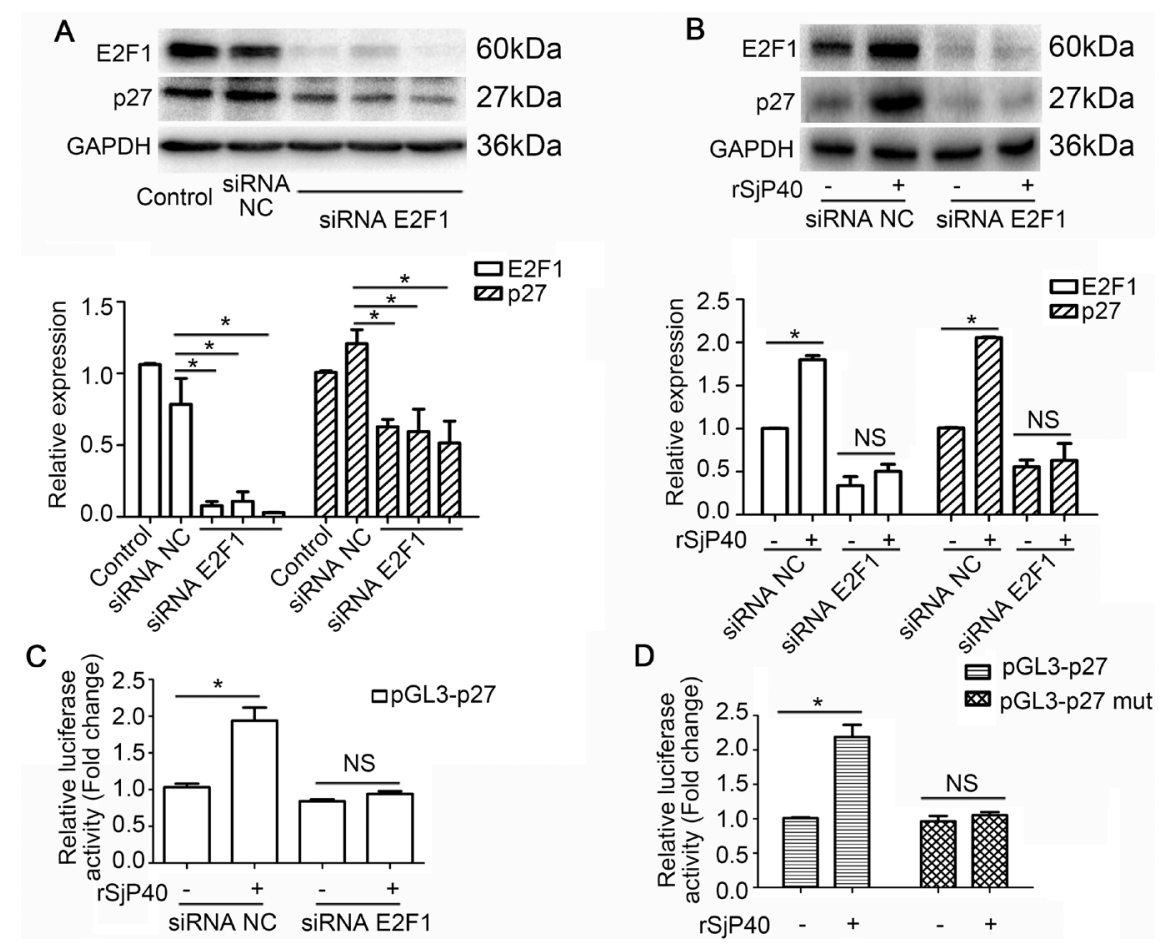

Figure 4: rSjP40 mediated enhancement of p27 promoter activity was related to E2F1 in LX-2 cells. (A) Relationship between E2F1 and p27 protein expression. ${ }^{*} P<0.05$, compared to siRNA NC- group. (B and $\mathbf{C}$ ) Western blot analysis and dual-luciferase reporter assay were conducted respectively to examine the effect of E2F1 on p27 expression in LX-2 cells treated with $\mathrm{rSjP} 40 .{ }^{*} P<0.05$, compared to rSjP40-siRNA NC+ group. NS represents $P>0.05$, between rSjP40-siRNA E2F1 + group and rSjP40 + siRNA E2F1 + group. (D) Dual-luciferase reporter assay was performed to observe the effect of rSjP40 on the activity of pGL3-p27 and pGL3-p27 mut in LX-2 cells. ${ }^{*} P<0.05$, compared to rSjP40-pGL3-p27+ group. NS represents $P>0.05$, between rSjP40-pGL3-p27 mut + group and rSjP40 + pGL3-p27 mut + group. The data are presented as the mean \pm SEM of at least three independent experiments. 
Although it is traditionally believed that schistosome eggs are the cause of liver fibrosis in schistosomiasis, Anthony et al. first demonstrated that eggs from $S$. mansoni could inhibit the activation of LX-2 cells directly, accompamied by an increased accumulation of lipid droplets in LX-2 cells [5]. They also reported that eggs from $S$. japonicum were able to exert an anti-fibrotic effect on LX-2 cells [6]. Based on their novel findings, we further demonstrated that SEA from $S$. japonicum may block liver fibrosis by inducing HSC apoptosis and senescence $[4,10]$. In addition, the anti-activating effect of SEA was confirmed both in HSCs activated in vivo and in vitro [4]. $\mathrm{P} 40$ protein from $S$. japonicum $(\mathrm{SjP} 40)$ and $S$. mansoni (SmP40) is the main component of SEA. SmP40 has been described as a mediator associated with reducing collagen deposition and fibrosis [19]. Recently, we also identified that $\mathrm{rSjP} 40$ could inhibit TGF- $\beta 1$-induced activation of LX-2 cells through the TGF- $\beta 1 /$ Smads pathway [13]. Interestingly, $\mathrm{rSjP} 40$ is different from SEA and could not induce cell apoptosis in LX-2 cells [20]. Importantly, we found that the anti-fibrotic effects of SEA and rSjP40 both manifested simultaneously with the induction of cell cycle arrest. As reported, SEA may induce cell cycle arrest at the G1 phase and inhibit HSC proliferation in vitro $[4,10]$. rSjP40 also can also induce arrest at the G1 phase and induce cell senescence in LX-2 cells [7]. Therefore, we speculated that SEA and rSjP40 may block liver fibrosis by arresting HSCs at the G1 phase, inhibiting HSC activation and inducing HSC senescence.

Previous studies showed that p27 protein could participate in the arrest process of the cell cycle and cell proliferation [8]. As Egozi et al. reported, p27 could negatively regulate the activity of protein kinase complex cyclin E-CDK2, thereby blocking cell cycle progression from $\mathrm{G} 1$ to $\mathrm{S}$ phase [21]. Eukaryotic translation initiation factor $3 \mathrm{a}(\mathrm{eIF} 3 \mathrm{a}) / \mathrm{p} 27$ pathway is considerably essential for TGF- $\beta 1$-induced cell proliferation in rats pulmonary fibroblasts. L-mimosine treatment could up-regulate p27 expression and attenuate pulmonary fibrosis [22]. In liver fibrosis, fluorofenidone, a pyridine agent, could alleviate liver fibrosis by inhibiting HSC activation and proliferation. The potential mechanism of this blocking

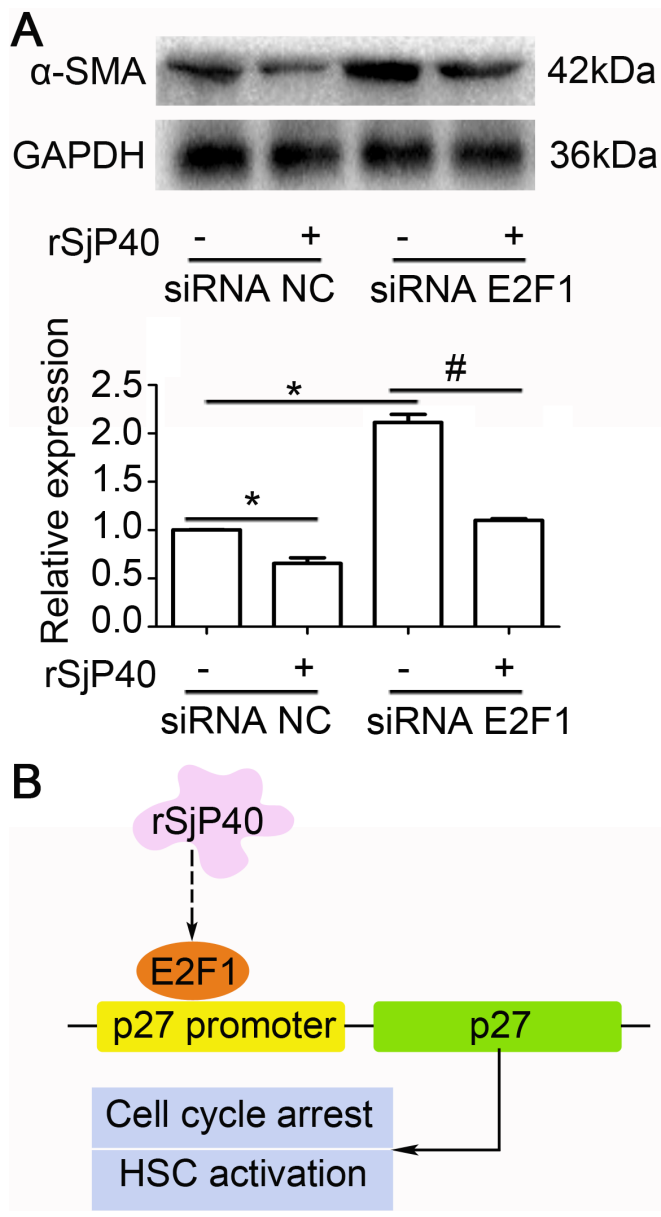

Figure 5: Inhibition of $\alpha-S M A$ expression mediated by rSjP40 was related to E2F1 in LX-2 cells. (A) Effect of E2F1 on $\alpha$-SMA expression in LX-2 cells treated with $\mathrm{rSjP} 40$ was detected by Western blot. $* P<0.05$, compared to rSjP40-siRNA NC+ group. ${ }^{\#} P<0.05$, compared to rSjP40-siRNA E2F1+ group. (B) Potential mechanism of rSjP40-mediated inhibition on activation of LX-2 cells was shown. rSjP40 protein may inhibit activation of LX-2 cells via a p27 and E2F1 dependent mechanism. 
effect of fluorofenidone may be attributed to its enhancive effect on p27 expression, which was associated with G0/G1 phase cell cycle arrest of fluorofenidone-treated HSCs [23]. In our previous studies, we also confirmed that SEA and rSjP40 could induce HSC senescence, which manifested as cell cycle arrest of HSCs, partly through the up-regulation of p27 expression [20, 24]. In this study, we further demonstrated that both SEA and rSjP40 could enhance the activity of $\mathrm{p} 27$ promoter.

It has been established that E2F1 is also involved in the regulation of cell cycle, and the $\mathrm{pRb} 2 / \mathrm{HDAC} 1 / \mathrm{E} 2 \mathrm{~F} 1$ complex may negatively regulate cell cycle regulatory proteins, including $\mathrm{Cdk} 2 / 4$ and cyclin D3/E [25]. The transcription factor E2F1 can bind to the $\mathrm{p} 27$ promoter and subsequently, enhance p27 expression [26]. In addition, a negative feedback mechanism was observed between p27 and E2F1, as inhibition of endogenous p27 expression could further promote E2F1 functions in H1299 cells through $\mathrm{pRb}$ [26]. In our study, we found that $\mathrm{rSjP} 40$ could induce p27 and E2F1 expression simultaneously. Further study demonstrated that the reduction of E2F1 by siRNA could block the rSj40-induced upregulation of p27 expression. Meanwhile, mutations in the E2F1 binding site of the p27 promoter could also block the rSjP40-induced up-regulation of p 27 promoter activity. However, siRNA of E2F1 could partially restore the effect of $\mathrm{rSjP} 40$ on the inhibition of $\alpha$-SMA expression and we speculated some other signals may exist in rSjP40-inhibited HSC activation.

In conclusion, we concluded that $\mathrm{rSjP} 40$ protein may enhance p 27 promoter activity in LX-2 cells via an E2F1-dependent mechanism, which may further induce HSC cell cycle arrest and partially inhibit the activation of HSCs (Figure 5B).

\section{MATERIALS AND METHODS}

\section{Cell culture and treatment}

LX-2 cells, a human HSC line, preserved in our lab [27], were cultured in Dulbecco's Modified Eagle's Medium (DMEM, Gibco, USA) supplemented with 10\% of fetal bovine serum (FBS), in a humidified atmosphere containing $5 \% \mathrm{CO}_{2}$ at $37^{\circ} \mathrm{C}$. LX-2 cells were inoculated into 6 well culture-plates and treated with $\mathrm{rSjP} 40$ at the concentration of $20 \mu \mathrm{g} / \mathrm{mL} . \mathrm{rSjP} 40$ was obtained as previously described [13].

\section{Bioinformatics analysis of p27 promoter and construction of plasmids containing p27 promoter sequence}

The sequence of the $\mathrm{p} 27$ promoter $(-1737 \mathrm{bp}$ to +24 bp), encompassing the ATG start site, was obtained from the National Center for Biotechnology Information (NCBI, http://www.ncbi.nlm.nih.gov/). Transcription factor binding sites were predicted using the JASPAR network tool software (http://jaspar.genereg.net/) and p-match network platform (http://www.gene-regulation.com/ pub/programs.html\#pmatch). To construct p27 promoter associated plasmids, the polymerase chain reaction (PCR) primers were designed (Table 1). Genomic DNA was extracted from LX-2 cells according to instructions for the QIAamp ${ }^{\circledR}$ DNA Micro Kit (Qiagen, Germany). Promoter fragments of p27 from -1737 bp to +24 bp was amplified from genomic DNA template, cloned into pGL3-basic vector (Promega, USA) and renamed as pGL3-p27. Then, four luciferase reporter plasmids containing truncated fragment of $\mathrm{p} 27$ promoter and one plasmid containing the mutant E2F1-binding site were generated separately using corresponding PCR primers shown in Table 1, and were named as pGL3-p27a, pGL3-p27b, pGL3-p27c, pGL3p27d and pGL3-p27 mut, respectively.

\section{Dual-luciferase reporter assay}

Reporter plasmids were transiently transfected into LX-2 cells according to the manufacturer's instructions for FuGENE Transfection Reagent (Promega, USA). After transfection for $12 \mathrm{~h}, \mathrm{rSjP} 40$ were added and LX-2 cells were cultured for another $24 \mathrm{~h}$. Then the cells were harvested for luciferase activity analysis on a luminometer following the instructions of the Dual-luciferase Reporter Assay system (Promega, USA).

\section{E2F1 interference experiment}

LX-2 cells were seeded in a 6 -well plate at $2 \times 10^{5}$ cells/well in $2 \mathrm{~mL}$ of medium without antibiotic/antimycotic and cultured to $70-90 \%$ confluency before transfection. Then $5 \mu \mathrm{L}$ of lipofectamine 2000 reagent (Invitrogen, USA) was diluted with $500 \mu \mathrm{L}$ of DMEM and incubated for $5 \mathrm{~min}$ at room temperature. Then, $5 \mu \mathrm{L}$ of siRNA E2F1 (Sigma, USA) or negative control siRNA (siRNA NC, Sigma, USA) were added to the lipofectamine 2000/medium mixture and incubated for $20 \mathrm{~min}$ at room temperature. Next, the mixture was added to $2 \mathrm{~mL}$ of cell culture media in the 6 -well plate. After transfection for $6 \mathrm{~h}$, the culture medium was discarded and replaced with medium containing rSjP40 at a concentration of $20 \mu \mathrm{g} / \mathrm{mL}$ for another $24 \mathrm{~h}$.

\section{Chromatin immunoprecipitation (ChIP)}

ChIP experiments were performed using SimpleChip Kit (Cell Signaling Technology, USA). Anti-E2F1 antibody was purchased from Santa Cruz Biotechnology (USA) and was used to precipitate the DNA-protein complex. Normal IgG provided in SimpleChip Kit was used as the negative control. Purified DNA obtained from SimpleChip Kit was then used as the template and PCR was conducted using two pairs of primers (Table 1), which were designed based on the different E2F1-binding sites of the p27 promoter. 
Table 1: Primers used in this study

\begin{tabular}{lll}
\hline primer & Sequence $\left(\mathbf{5}^{\prime} \rightarrow \mathbf{3}^{\prime}\right)$ & Purpose \\
\hline p27 $\mathrm{F}^{1}$ & GCCCTGCTCATCGTCCTACTTTAC & pGL3-p27 \\
p27a F & CGTTCGCTTTGGCTTCTTCCCT & pGL3-p27a \\
p27b F & CGGTCCTCTGGTCCAGGTCC & pGL3-p27b \\
p27c F & CGCCGCAACCAATGGATCTC & pGL3-p27c \\
p27d F & TCGCCAGTCCATTTGATCAGC & pGL3-p27d \\
p27 R & GTTAGACACTCGCACGTTTGACATC & Reporters \\
p27-mut F1 & GCCCTGCTCATCGTCCTACTTTACCTTC & pGL3-p27 mut \\
p27-mut R1 & GAGTGTGCGATGTAGATACAACAGCTCCTTCC & pGL3-p27 mut \\
p27-mut F2 & TGTATCTACATCGCACACTCAGGTAGAGGAAA & pGL3-p27 mut \\
p27-mut R2 & GTTAGACACTCGCACGTTTGACATC & pGL3-p27 mut \\
E2F1(a) F & GTTGGAGCAGTGAAATCTGGTGAG & ChIP \\
E2F1(a) R & AACTCGTCCCTTTCTACTTTTCTG & ChIP \\
E2F1(b) F & GAACCATTGCCCACTGCCTC & ChIP \\
E2F1(b) R & ACCTCGTGGTCTGCGGGGGA & ChIP \\
\hline
\end{tabular}

${ }^{1} \mathrm{~F}$ : Forward.

${ }^{2} \mathrm{R}$ : Reverse.

${ }^{3}$ Reporters: pGL3-p27, pGL3-p27a, pGL3-p27b, pGL3-p27c, pGL3-p27d.

\section{Western blot}

Whole protein samples were obtained from lysates of LX-2 cells in RIPA buffer (Beyotime, China) and subjected to $10 \%$ sodium dodecyl sulfate-polyacrylamide gel electrophoresis (SDS-PAGE). The proteins were then transferred to $0.45 \mu \mathrm{m}$ polyvinylidene fluoride (PVDF) membranes (Merck, Germany). The membranes were then incubated at $4{ }^{\circ} \mathrm{C}$ for $12 \mathrm{~h}$ with primary antibodies including p27 (Santa Cruz, USA), E2F1 (Santa Cruz, USA), $\alpha$-SMA (Santa Cruz, USA) and GAPDH (Goodhere, China). Then, the membranes were washed and incubated with horseradish peroxidase (HRP)- conjugated secondary antibodies (Santa Cruz Biotechnology, USA) and visualized with ECL-chemiluminescent kit (Merck, Germany).

\section{Statistical analysis}

The data are presented as the mean \pm SEM of at least three independent experiments. Data were analyzed using one-way ANOVA method or Independent Samples $T$-test in SPSS 15.0. Differences were considered significant if $P<0.05$.

\section{ACKNOWLEDGMENTS AND FUNDING}

This work was supported by National Natural Science Foundation of China (Grant Numbers 81471975, $81501763,81401683,81171589)$, the Jiangsu provincial Natural Science Foundation (Grant Number BK20140435), the Priority Academic Program Development of Jiangsu Higher Education Institutions (PAPD) and the Graduate Student Research and Innovation Program of Nantong University (Grant Number YKC15054).

\section{CONFLICTS OF INTEREST}

There are no potential conflicts of interest to declare.

\section{REFERENCES}

1. Wang N, Xu Q, Tan HY, Hong M, Li S, Yuen MF, Feng Y. Berberine Inhibition of Fibrogenesis in a Rat Model of Liver Fibrosis and in Hepatic Stellate Cells. Evid Based Complement Alternat Med. 2016; 2016:8762345.

2. Yang JH, Kim SC, Kim KM, Jang CH, Cho SS, Kim SJ, $\mathrm{Ku} \mathrm{SK}$, Cho IJ, Ki SH. Isorhamnetin attenuates liver fibrosis by inhibiting TGF-beta/Smad signaling and relieving oxidative stress. Eur J Pharmacol. 2016; 783:92-102.

3. Andrade ZA. Schistosomiasis and liver fibrosis. Parasite Immunol. 2009; 31:656-663.

4. Duan Y, Gu X, Zhu D, Sun W, Chen J, Feng J, Song K, Xu F, $\mathrm{He} \mathrm{X}$. Schistosoma japonicum soluble egg antigens induce apoptosis and inhibit activation of hepatic stellate cells: a possible molecular mechanism. Int J Parasitol. 2014; 44:217-224.

5. Anthony B, Mathieson W, de Castro-Borges W, Allen J. Schistosoma mansoni: egg-induced downregulation of hepatic stellate cell activation and fibrogenesis. Exp Parasitol. 2010; 124:409-420.

6. Anthony BJ, James KR, Gobert GN, Ramm GA, McManus DP. Schistosomajaponicum Eggs Induce a Proinflammatory, Anti-Fibrogenic Phenotype in Hepatic Stellate Cells. Plos One. 2013; 8:e68479.

7. Chen J, Xu T, Zhu D, Wang J, Huang C, Lyu L, Hu B, Sun W, Duan Y. Egg antigen p40 of Schistosoma japonicum promotes senescence in activated hepatic stellate cells by activation of the STAT3/p53/p21 pathway. Cell Death Dis. 2016; 7:e2315. 
8. Bedir R, Gucer H, Sehitoglu I, Yurdakul C, Bagci P, Ustuner P. The Role of p16, p21, p27, p53 and Ki-67 Expression in the Differential Diagnosis of Cutaneous Squamous Cell Carcinomas and Keratoacanthomas: An Immunohistochemical Study. Balkan Med J. 2016; 33:121-127.

9. Li A, Wang J, Wu M, Zhang X, Zhang H. The inhibition of activated hepatic stellate cells proliferation by arctigenin through G0/G1 phase cell cycle arrest: persistent p27(Kip1) induction by interfering with PI3K/Akt/FOXO3a signaling pathway. Eur J Pharmacol. 2015; 747:71-87.

10. Chen J, Pan J, Wang J, Song K, Zhu D, Huang C, Duan Y. Soluble egg antigens of Schistosoma japonicum induce senescence in activated hepatic stellate cells by activation of the STAT3/p53/p21 pathway. Sci Rep. 2016; 6:30957.

11. Ye D, Luo H, Lai Z, Zou L, Zhu L, Mao J, Jacob T, Ye W, Wang L, Chen L. ClC-3 Chloride Channel Proteins Regulate the Cell Cycle by Up-regulating cyclin D1-CDK4/6 through Suppressing p21/p27 Expression in Nasopharyngeal Carcinoma Cells. Sci Rep. 2016; 6:30276.

12. Zhong S, Li YG, Ji DF, Lin TB, Lv ZQ. Protocatechualdehyde Induces S-Phase Arrest and Apoptosis by Stimulating the p27(KIP1)-Cyclin A/D1CDK2 and Mitochondrial Apoptotic Pathways in HT-29 Cells. Molecules. 2016; 21.

13. Sun X, Zhang L, Wang J, Chen J, Zhu D, Shen P, He X, Pan J, Peng W, Duan Y. Schistosoma japonicum protein SjP40 inhibits TGF-beta1-induced activation of hepatic stellate cells. Parasitol Res. 2015; 114:4251-4257.

14. Pearce EJ, MacDonald AS. The immunobiology of schistosomiasis. Nat Rev Immunol. 2002; 2:499-511.

15. Anthony BJ, Ramm GA, McManus DP. Role of resident liver cells in the pathogenesis of schistosomiasis. Trends Parasitol. 2012; 28:572-579.

16. Wang J, Chu ES, Chen HY, Man K, Go MY, Huang XR, Lan HY, Sung JJ, Yu J. microRNA-29b prevents liver fibrosis by attenuating hepatic stellate cell activation and inducing apoptosis through targeting PI3K/AKT pathway. Oncotarget. 2015; 6:7325-7338. doi: 10.18632/ oncotarget.2621.

17. An J, Zheng L, Xie S, Yin F, Huo X, Guo J, Zhang X. Regulatory Effects and Mechanism of Adenovirus-Mediated PTEN Gene on Hepatic Stellate Cells. Dig Dis Sci. 2016; 61:1107-1120.

18. Bohanon FJ, Wang X, Graham BM, Prasai A, Vasudevan SJ, Ding C, Ding Y, Radhakrishnan GL, Rastellini C, Zhou J,
Radhakrishnan RS. Enhanced anti-fibrogenic effects of novel oridonin derivative CYD0692 in hepatic stellate cells. Mol Cell Biochem. 2015; 410:293-300.

19. Abouel-Nour MF, Lotfy M, Attallah AM, Doughty BL. Schistosoma mansoni major egg antigen Smp40: molecular modeling and potential immunoreactivity for anti-pathology vaccine development. Mem Inst Oswaldo Cruz. 2006; 101:365-372.

20. Xu T, Chen J, Zhu D, Chen L, Wang J, Sun X, Hu B, Duan Y. Egg antigen p40 of Schistosoma japonicum promotes senescence in activated hepatic stellate cells via SKP2/P27 signaling pathway. Sci Rep. 2017; 7:275.

21. Egozi D, Shapira M, Paor G, Ben-Izhak O, Skorecki K, Hershko DD. Regulation of the cell cycle inhibitor p27 and its ubiquitin ligase Skp2 in differentiation of human embryonic stem cells. FASEB J. 2007; 21:2807-2817.

22. Li XW, Hu CP, Li YJ, Gao YX, Wang XM, Yang JR. Inhibitory effect of 1-mimosine on bleomycin-induced pulmonary fibrosis in rats: Role of eIF3a and p27. Int Immunopharmacol. 2015; 27:53-64.

23. Peng Y, Yang H, Wang N, Ouyang Y, Yi Y, Liao L, Shen H, Hu G, Wang Z, Tao L. Fluorofenidone attenuates hepatic fibrosis by suppressing the proliferation and activation of hepatic stellate cells. Am J Physiol Gastrointest Liver Physiol. 2014; 306:G253-263.

24. Duan Y, Pan J, Chen J, Zhu D, Wang J, Sun X, Chen L, $\mathrm{Wu}$ L. Soluble Egg Antigens of Schistosoma japonicum Induce Senescence of Activated Hepatic Stellate Cells by Activation of the FoxO3a/SKP2/P27 Pathway. PLoS Negl Trop Dis. 2016; 10:e005268.

25. Chang CC, Sue YM, Yang NJ, Lee YH, Juan SH. 3-Methylcholanthrene, an AhR agonist, caused cell-cycle arrest by histone deacetylation through a RhoA-dependent recruitment of HDAC1 and $\mathrm{pRb} 2$ to E2F1 complex. Plos One. 2014; 9:e92793.

26. Wang C, Hou X, Mohapatra S, Ma Y, Cress WD, Pledger WJ, Chen J. Activation of p27Kip1 Expression by E2F1. A negative feedback mechanism. J Biol Chem. 2005; 280:12339-12343.

27. Wang J, Xu F, Zhu D, Duan Y, Chen J, Sun X, He X, Li P, Sun W, Feng J. Schistosoma japonicum soluble egg antigens facilitate hepatic stellate cell apoptosis by downregulating Akt expression and upregulating p53 and DR5 expression. PLoS Negl Trop Dis. 2014; 8:e3106. 